\title{
q-Análogos em Corpos Finitos: Definição, Propriedades Algébricas e Aplicação em Geradores de Números Pseudo-Aleatórios
}

\author{
Carlos E. C. Souza, Ravi B. D. Figueiredo, Daniel P. B. Chaves e Cecilio Pimentel
}

\begin{abstract}
Resumo-Neste trabalho é definido o $q$-análogo do corpo finito $\mathbb{Z}_{p}$, denotado por $\left[\mathbb{Z}_{p}\right]_{q}$. São derivadas várias propriedades de $\left[\mathbb{Z}_{p}\right]_{q}$ e são estabelecidas as condições necessárias para que o mapeamento entre $\mathbb{Z}_{p}$ e $\left[\mathbb{Z}_{p}\right]_{q}$ seja uma bijeção, definindo uma permutação. Os q-análogos em corpos finitos são empregados para construir geradores de números pseudo-aleatórios (PRNG, pseudo-random number generator) e suas propriedades estatísticas são analisadas. Simulações computacionais utliizando a suíte estatística NIST evidenciam que os geradores propostos geram sequências com boas propriedades estatísticas e taxa máxima de geração de bits.
\end{abstract}

Palavras-Chave- $q$-Análogos, corpos finitos, teoria dos números, geradores de números pseudo-aleatórios.

Abstract-In this work we define the $q$-analog of the finite field $\mathbb{Z}_{p}$, denoted as $\left[\mathbb{Z}_{p}\right]_{q}$. We derive several properties of $\left[\mathbb{Z}_{p}\right]_{q}$ and investigate the necessary conditions to obtain a bijection between $\mathbb{Z}_{p}$ and $\left[\mathbb{Z}_{p}\right]_{q}$, defining a permutation. We employ the $q$-analogs in finite fields to design a pseudo-random number generator (PRNG) and its statistical properties are analyzed. Computer simulations show that the proposed pseudo-random number generator generates binary sequences with good statistical properties and maximal bit rate generation.

Keywords- $q$-Analogs, finite fields, number theory, pseudorandom number generators.

\section{INTRODUÇÃO}

$q$-Análogos, também denominados $q$-deformações, são generalizações de objetos matemáticos tais como múmeros e funções, parametrizados em função de um parâmetro $q$, de forma que no limite $q \rightarrow 1$ o objeto original é recuperado [1]. Inicialmente, os $q$-análogos foram definidos sobre o conjunto dos números inteiros no contexto de combinatória [2]. Trabalhos recentes propõem a generalização do conceito de $q$-análogos para conjuntos numéricos mais gerais, como os números racionais e reais [3], [4].

Em outro contexto, alguns trabalhos vêm considerando a introdução da $q$-deformação em mapas caóticos reais, com o objetivo de investigar o efeito da $q$-deformação na dinâmica caótica [5], [6], [7], [8]. A introdução da $q$-deformação, portanto, incorpora um grau de liberdade adicional na dinâmica complexa gerada por mapas caóticos, sendo as $q$-deformações potenciais generalizações para agregar uma camada adicional de complexidade à dinâmica caótica. Além disso, o conceito de caos discreto foi desenvolvido em [9] e mapas caóticos

Os autores são do Departmento de Eletrônica e Sistemas, Universidade Federal de Pernambuco, Recife-PE, e-mails: \{carlos.ecsouza, ravi.figueiredo, daniel.chaves, cecilio.pimentel\}@ufpe.br. Este trabalho foi parcialmente financiado pelo CNPq, pela CAPES e pela FACEPE. sobre conjuntos discretos vêm sendo aplicados com sucesso no projeto de geradores de números pseudo-aleatórios (PRNG, pseudo-random number generator) [10]. Estes mapas têm a vantagem de utilizar operações de ponto fixo, diminuindo o efeito de erros sistemáticos decorrentes de processos de quantização e arredondamentos característicos de sistemas definidos sobre os números reais [11].

Para que a camada adicional de complexidade incorporada pela deformação dos $q$-análogos possa ser aplicada em sistemas definidos em conjuntos discretos, é necessário estabelecer o conceito de $q$-deformação nesses conjuntos, como por exemplo, corpos finitos. Até onde chega o conhecimento dos autores, o conceito de $q$-análogos em corpos finitos ainda não está definido na literatura.

Neste trabalho é definido o $q$-análogo do corpo finito $\mathbb{Z}_{p}$, o conjunto das classes de equivalência dos números inteiros módulo $p$, em que $p$ é um número primo. A versão $q$ deformada de $\mathbb{Z}_{p}$ é denotada por $\left[\mathbb{Z}_{p}\right]_{q}$. São investigadas algumas propriedades algébricas de $\left[\mathbb{Z}_{p}\right]_{q}$ e é demonstrado que quando $q$ é um gerador do grupo multiplicativo associado a $\mathbb{Z}_{p}$, o mapeamento entre $\mathbb{Z}_{p}$ e $\left[\mathbb{Z}_{p}\right]_{q}$ é uma bijeção, ou seja, $\left[\mathbb{Z}_{p}\right]_{q}$ é uma permutação de $\mathbb{Z}_{p}$. Esta permutação é empregada no projeto de um PRNG e as propriedades estatísticas das sequências binárias geradas são analisadas com a suíte estatística NIST [12]. O PRNG proposto é aprovado em todos os testes da suíte NIST usando uma taxa máxima de geração de bits, ou seja, todos os bits da representação binária são utilizados para gerar a sequência binária do PRNG. Como geralmente os geradores propostos na literatura não empregam todos os bits da representação binária, o PRNG proposto além de apresentar boas propriedades estatísticas possui como vantagem uma alta taxa de geração de bits.

O restante deste trabalho está dividido conforme detalhado a seguir. Na Seção II é feita uma breve revisão de conceitos fundamentais de $q$-análogos e corpos finitos. A definição e investigação de propriedades algébricas dos $q$-análogos em corpos finitos é apresentada na Seção III. Na Seção IV é feita uma aplicação de $q$-análogos em corpos finitos no projeto de geradores de números pseudo-aleatórios e suas propriedades estatísticas são analisadas com a suíte NIST [12]. Por fim, na Seção V são apresentadas as considerações finais.

\section{PRELIMINARES}

Nesta seção são revisadas algumas definições e propriedades elementares de $q$-análogos. Também é feita uma breve 
introdução ao conceito de corpos finitos, sendo apresentadas propriedades necessárias para a compreensão do restante deste trabalho. Mais detalhes sobre $q$-análogos são encontrados em [1], [2] e sobre corpos finitos em [13].

Seja $n$ um inteiro não negativo e seja $q \in \mathbb{R}$. O $q$-análogo de $n$ (também denominado $q$-deformação de $n$ ), denotado por $[n]_{q}$, é definido por

$$
[n]_{q} \triangleq \frac{1-q^{n}}{1-q}
$$

Alternativamente, $[n]_{q}$ pode ser escrito como a série de potências em $q$

$$
[n]_{q}=1+q+q^{2}+\cdots+q^{n-1} .
$$

O $q$-análogo de zero é definido, por convenção, por $[0]_{q}=0$. É evidente, a partir de (1), que $[1]_{q}=1$. No limite $q \rightarrow 1$ temos que $\lim _{q \rightarrow 1}[n]_{q} \triangleq[n]_{q \rightarrow 1}=n$. q-Análogos também podem ser definidos pela relação de recorrência

$$
[n+1]_{q}=q[n]_{q}+1 \text {. }
$$

Seja $p$ um número primo e seja $\mathbb{Z}_{p}=\{0,1,2, \ldots, p-1\}$ o conjunto das classes de equivalência dos inteiros módulo p. O conjunto $\mathbb{Z}_{p}$ com as operações de adição e multiplicação definem o corpo finito $\left(\mathbb{Z}_{p},+, \times\right)$ de característica $p$, também denotado por $\operatorname{GF}(p)$ (Galois Field). Quando não houver ambiguidade, o corpo finito $\left(\mathbb{Z}_{p},+, \times\right)$ será referido como o corpo finito $\mathbb{Z}_{p}$. Dado o conjunto $\mathbb{Z}_{p}$, o subconjunto $\mathbb{Z}_{p}-\{0\}=\{1,2, \ldots, p-1\}$ é um grupo Abeliano de ordem $p-1$ sob a operação de multiplicação em $\mathbb{Z}_{p}$ e será denotado por $G=\left(\mathbb{Z}_{p}-\{0\}, \times\right)$. O grupo $G$ é um grupo cíclico e um gerador de $G$ é um elemento $g \in G$ tal que qualquer elemento $n \in G$ é dado por uma potência de $g$, ou seja, $G=\langle g\rangle=\left\{1, g, g^{2}, \ldots, g^{p-2}\right\}$. O número de geradores distintos de um grupo cíclico com ordem $l$ é dado pela função phi de Euler $\phi(l)$ [14]. Como $G$ possui ordem $p-1$, o número de geradores distintos de $G$ é $\phi(p-1)$.

\section{III. $q$-AnÁlogos EM CORPOS Finitos}

Nesta seção é definido o $q$-análogo em $\mathbb{Z}_{p}$. São derivadas várias propriedades algébricas dos $q$-análogos em $\mathbb{Z}_{p}$ e estabelecidas as condições necessárias para que a $q$-deformação seja uma operação de permutação. No restante do artigo, todas as operações são definidas módulo $p$.

Definição 1. Seja o corpo finito $\mathbb{Z}_{p}$ e seja $q \in \mathbb{Z}_{p}, q \neq 0$. $O$ $q$-análogo de $n \in \mathbb{Z}_{p}, n \neq 0$ é definido por

$$
[n]_{q} \triangleq \frac{q^{n}-1}{q-1}(\bmod p)
$$

e o q-análogo de $n=0$ é definido por

$$
\left\{\begin{array}{l}
{[0]_{1} \triangleq 0} \\
{[0]_{q} \triangleq(1-q)^{-1}(\bmod p), q \neq 1}
\end{array}\right.
$$

em que $(\cdot)^{-1}$ denota o inverso multiplicativo de (.) em $\mathbb{Z}_{p}$.

Similarmente ao caso dos $q$-análogos clássicos, os $q$ análogos definidos em corpos finitos podem ser escritos como uma série de potências em $q$

$$
[n]_{q}=\left(1+q+q^{2}+\cdots+q^{n-1}\right)(\bmod p) .
$$

Definição 2. Seja $q \in \mathbb{Z}_{p}, q \neq 0$. $O$ q-análogo do conjunto $\mathbb{Z}_{p}$ é definido pelo conjunto $\left[\mathbb{Z}_{p}\right]_{q} \triangleq\left\{[0]_{q},[1]_{q},[2]_{q}, \ldots,[p-1]_{q}\right\}$.

Quando $q=1$ o mapeamento entre $\mathbb{Z}_{p}$ e $\left[\mathbb{Z}_{p}\right]_{q}$ é a transformação identidade, conforme demonstrado na proposição a seguir.

Proposição 1. $[n]_{1} \equiv n(\bmod p), \forall n \in \mathbb{Z}_{p}$.

Demonstração: Usando (6) temos que $[n]_{1}=1+1^{1}+1^{2}+$ $\cdots+1^{n-1} \equiv n(\bmod p)$, pois $n<p, \forall n \in \mathbb{Z}_{p}$.

A proposição seguinte aborda um ponto importante no comportamento de mapas iterativos e sequências, a análise de pontos fixos. Da definição da $q$-deformação é simples verificar que 1 é um ponto fixo para qualquer valor de $q$.

Proposição 2. $[1]_{q} \equiv 1(\bmod p), \forall q \in \mathbb{Z}_{p}, q \neq 0$.

Demonstração: Segue diretamente de (4).

Enunciamos agora um teorema sobre a $q$-deformação de zero, afirmando que esta nunca pode ser igual a zero, ou seja, zero nunca é ponto fixo da $q$-deformação.

Teorema 1. $[0]_{q} \neq 0, \forall q \neq 1$.

Demonstração: Suponha que $[0]_{q} \equiv 0(\bmod p)$, então segue de (5) que $(1-q)^{-1} \equiv 0(\bmod p)$ e portanto $0 \times(1-q) \equiv$ $1(\bmod p)$, o que é uma contradição.

A partir de (6), segue que $[n]_{q}, n \geq 1$ pode ser alternativamente definido pela relação de recorrência

$$
[n+1]_{q}=\left(q[n]_{q}+1\right)(\bmod p)
$$

enquanto $[0]_{q}$ é definido separadamente por (5). Desta forma, para gerar o conjunto $\left[\mathbb{Z}_{p}\right]_{q}$ calculamos inicialmente $[0]_{q}$ e em seguida determinamos o restante dos $q$-análogos de forma iterativa a partir de $[1]_{q}=1$. Neste caso, a aplicação iterativa da $q$-deformação gera uma dinâmica iterativa discreta sobre $\mathbb{Z}_{p}$ gerada por (7) que está associada com a geração de sequências numéricas definidas em $\mathbb{Z}_{p}$.

Definição 3. $O$ q-análogo inverso de $[n]_{q} \in\left[\mathbb{Z}_{p}\right]_{q}$, isto é, $n \in \mathbb{Z}_{p}$, é definido por

$$
q^{n}=1+(q-1)[n]_{q}(\bmod p) .
$$

Observe que (8) pode ser escrita como

$$
n=\log _{q}\left(1+(q-1)[n]_{q}\right)(\bmod p)
$$

em que $\log _{q}(\cdot)$ é o logaritmo discreto de $(\cdot)$ na base $q$ no conjunto $\mathbb{Z}_{p}$. Para a demonstração do resultado principal deste trabalho, o Teorema 2, são necessários três lemas, os quais são enunciados a seguir.

Lema 1. Dados $n \neq 0$ e $q \neq 1$, então $[n]_{q} \equiv 0(\bmod p)$ se, $e$ somente se, $q^{n} \equiv 1(\bmod p)$.

Demonstração: Suponha que $[n]_{q} \equiv 0(\bmod p)$, portanto segue da Eq. (8) que $q^{n} \equiv 1(\bmod p)$. Agora, suponha que $q^{n} \equiv$ $1(\bmod p)$, então aplicando a Eq. (8) obtemos $(q-1)[n]_{q} \equiv$ $0(\bmod p)$. Consequentemente, $p$ divide $(q-1)$ ou $p$ divide $\left[n_{q}\right]$. Como $q-1<p$, então $q-1$ não possui fatores $p$ e portanto $p$ necessariamente divide $[n]_{q}$. Desta forma, concluímos que $[n]_{q} \equiv 0(\bmod p)$. 
O lema seguinte mostra que $p-1$ sempre é $q$-deformado em zero, para qualquer valor de $q \neq 1$.

Lema 2. $[p-1]_{q} \equiv 0(\bmod p), \forall q \in \mathbb{Z}_{p}, q \neq 1$. Demonstração: Do Lema $1,[p-1]_{q} \equiv 0(\bmod p)$ se, e somente se, $q^{p-1} \equiv 1(\bmod p)$. Agora, observe que $q^{p-1} \equiv 1(\bmod p)$ é verdadeiro para todo $q \neq 1$ pelo pequeno teorema de Fermat [14].

O próximo lema estabelece que existe um e apenas um elemento em $\mathbb{Z}_{p}$ que é $q$-deformado em zero quando $q$ é um gerador do grupo multiplicativo $G$.

Lema 3. Se q é um gerador de $G$, existe um único $n \in \mathbb{Z}_{p}$ com $n \neq 0$ tal que $[n]_{q} \equiv 0(\bmod p)$.

Demonstração: Suponha que existem $n, m \in \mathbb{Z}_{p}$ tais que $[n]_{q} \equiv 0(\bmod p) e[m]_{q} \equiv 0(\bmod p)$. Portanto, do Lema 1 , segue que $q^{n} \equiv 1(\bmod p)$ e $q^{m} \equiv 1(\bmod p)$ e consequentemente $q^{m} \equiv q^{n}(\bmod p)$. Como $q$ é gerador de $G$, as potências de q são necessariamente distintas, logo $q^{m} \equiv q^{n}(\bmod p)$ é verdadeiro se, e somente se, $n=m$.

De posse dos três lemas anteriores, podemos enunciar o resultado principal sobre a $q$-deformação em corpos finitos, que associa uma bijeção entre $\mathbb{Z}_{p}$ e $\left[\mathbb{Z}_{p}\right]_{q}$ com o parâmetro de deformação $q$, ou seja, a $q$-deformação define uma permutação sobre $\mathbb{Z}_{p}$.

Teorema 2. Se q é um gerador de $G$, o mapeamento entre $\mathbb{Z}_{p}$ $e\left[\mathbb{Z}_{p}\right]_{q}$ é uma bijeção.

Demonstração: Sejam $n, m \in \mathbb{Z}_{p}$ com $n \neq m$ e tais que $[n]_{q} \equiv[m]_{q}(\bmod p)$. Vamos assumir, sem perda de generalidade, que a representação de $[n]_{q}$ em potências de $q$ tem mais termos que a represenção de $[\mathrm{m}]_{q}$, logo $[n]_{q}-[\mathrm{m}]_{q}=$ $q^{m}+q^{m+1}+\cdots+q^{n-1}$. Como $[n]_{q} \equiv[m]_{q}(\bmod p)$, então segue que $q^{m}+q^{m+1}+\cdots+q^{n-1} \equiv 0(\bmod p)$, que pode ser escrito como $q^{m}\left(1+q+q^{2}+\cdots+q^{n-m-1}\right) \equiv 0(\bmod p)$, ou equivalentemente $q^{m}[n-m]_{q} \equiv 0(\bmod p)$. Desta forma, temos que ou $p$ divide $q^{m}$ ou $p$ divide $[n-m]_{q}$. Como $q<p$, então $q^{m}$ não possui fatores $p$ e consequentemente $p$ não divide $q^{m}$. Segue que $p$ necessariamente divide $[n-m]_{q} e$ portanto $[n-m]_{q} \equiv 0(\bmod p)$. Do Lema 2 temos que $[n-m]_{q} \equiv[p-1]_{q} \equiv 0(\bmod p)$. Como q é um gerador de $G$, pelo Lema 3 , existe um único elemento em $\mathbb{Z}_{p}$ com que é deformado em zero, que é o próprio $p-1$, portanto $n-m=p-1$. Agora, observe que o único par possivel $(n, m)$ em $\mathbb{Z}_{p}$ que satisfaz esta condição é o par $(n, m)=(p-1,0)$ e obviamente $[p-1]_{q} \not \equiv[0]_{p}(\bmod p)$. Como assumimos inicialmente que $[n]_{q} \equiv[m]_{q}(\bmod p)$, chegamos a uma contradição, concluindo, portanto, a demonstração.

O Teorema 2 garante que se $q$ for um gerador de $G$, o conjunto $\left[\mathbb{Z}_{p}\right]_{q}$ é uma permutação do conjunto $\mathbb{Z}_{p}$. Neste caso, o $q$-análogo de $\mathbb{Z}_{p}$ é interpretado como um elemento do grupo de permutações $S_{p}$. Obviamente, o número de geradores de $G$ é menor que $p$, e portanto o conjunto de todas os $q$-análogos gerados por geradores de $G$ é um subconjunto de $S_{p}$.

\section{AplicaÇão: Geradores de Números PSEUDOALEATÓRIOS}

Nesta seção, utilizamos a dinâmica discreta gerada pela aplicação iterativa da $q$-deformação para projetar um PRNG e analisamos suas propriedades estatísticas utilizando a suíte estatística NIST.

A $q$-deformação como aplicação iterativa define um mapa discreto com uma estrutura linear sobre $\mathbb{Z}_{p}$. Consideramos que a sequência inicial é dada por $\{0,1,2, \ldots, p-1\}$. Em seguida, calculamos a sequência $q$-deformada a partir de (6), observando que a deformação de zero é definida particularmente por (5). Esta sequência possui estrutura similar às sequências geradas pela classe de geradores de números pseudo-aleatórios lineares denominados LCG (linear congruential generator), que são geradores utilizados em diversas plataformas pela simplicidade da implementação e boas propriedades estatísticas.

Como a $q$-deformação em corpos finitos está associada a uma dinâmica discreta em $\mathbb{Z}_{p}$, os elementos de $[\mathbb{Z}]_{p}$ podem ser intrepretados como uma sequência obtida pela permutação dos elementos em $\mathbb{Z}_{p}$. Desta forma, sendo a $q$-deformação uma permutação (se $q$ é um gerador de $G$ ), o período das sequências geradas pela $q$-deformação é igual à cardinalidade do conjunto $\mathbb{Z}_{p}$. Para a implementação de um PRNG, os elementos destas sequências são transformados em suas representações binárias. Em seguida, é extraído um subconjunto de bits desta representação, e os bits extraídos são concatenados para formar a sequência binária gerada pelo PRNG. Isto define uma taxa $r=k / b$, em que $k$ é o número de bits extraídos de cada elemento e $b$ é o número de bits na representação binária.

Para analisar as propriedades estatísticas das sequências binárias geradas, utilizamos a suíte estatística NIST versão SP800-22 [12]. Cada teste do NIST é realizado com um nível de confiança $\alpha=0,01$, sendo este o valor recomendado em [12]. Os testes são realizados com um conjunto de 200 subsequências binárias, cada uma com comprimento $10^{6}$. Para isto, inicialmente escolhemos os parâmetros $p$ e $q$ para definir a $q$-deformação. Em particular, utilizamos o primo de Mersenne $p=2^{31}-1$ e $q=16807$. Desta forma, cada elemento $q$-deformado de $\mathbb{Z}_{p}$ é representado com 31 bits. Os testes realizados indicam que PRNG proposto é aprovado na bateria de testes NIST quando utiliza-se todos os bits da representação binária, isto é, obtém-se a taxa máxima $r=1{ }^{1}$ Esta característica é uma vantagem em relação a geradores usuais da literatura com taxa $r<1$ [15], [16], [10].

A Tabela I mostra os resultados obtidos com a suíte NIST para o PRNG proposto com os parâmetros indicados. Para critério de comparação, também incluímos o resultado do teste NIST para o PRNG baseado no mapa de Arnold proposto em [10]. Na tabela é listado cada um dos testes do NIST e a proporção de sequências aprovadas em cada teste. Quando um teste é composto de múltiplas instâncias (estes são indicados por um *), apresentamos apenas o menor valor obtido. Para os parâmetros considerados, a proporção mínima de sequências que devem obter sucesso no teste é 0,965 . O PRNG proposto

\footnotetext{
${ }^{1}$ Para gerar as sequências binárias de entrada do teste NIST calculamos a permutação de $\{0,1,2, \ldots, N\}$ para um valor suficientemente grande de $N$ para gerar 200 milhões de bits.
} 
TABELA I

PROPORÇÕES OBTIDAS COM AS SUÍTE ESTATÍSTICA NIST PARA AS SEQUÊNCIAS BINÁRIAS GERADAS PELO PRNG PROPOSTO E PARA O PRNG PROPOSTO EM [10]. O VALOR MÍNIMO PARA APROVAÇÃO É 0,965. NOS TESTES MÚLTIPLOS (INDICADOS POR *) É APRESENTADO APENAS O VALOR MÍNIMO.

\begin{tabular}{|c||c|c|}
\hline Teste Estatístico & $q$-Análogos & Arnold \\
\hline \hline Frequency & 0,985 & 0,995 \\
\hline Block Frequency & 0,995 & 0,985 \\
\hline Cumulative Sums* & 0,985 & 0,99 \\
\hline Runs & 0,995 & 0,985 \\
\hline Longest Run & 0,98 & 0,99 \\
\hline Rank & 0,995 & 1 \\
\hline FFT & 0,97 & 0,985 \\
\hline Non Overlapping Template* & 0,97 & 0,965 \\
\hline Overlapping Template & 0,985 & 0,985 \\
\hline Universal & 0,98 & 0,97 \\
\hline Approximate Entropy & 0,99 & 0,99 \\
\hline Random Excursions* & 0,974 & 0,976 \\
\hline Random Excursions Variant* & 0,982 & 0,968 \\
\hline Serial* & 0,98 & 0,985 \\
\hline Linear Complexity & 0,985 & 0,985 \\
\hline
\end{tabular}

em [10] utiliza o mapa de Arnold discreto definido sobre o anel de inteiros $\mathbb{Z}_{3^{m}}$. Para $m=20$ podemos utilizar uma representação de 32 bits para cada elemento gerado pela aplicação iterativa do mapa de Arnold discreto. Em particular, o PRNG proposto em [10] tem taxa máxima $r=0,75$ para obtenção de aprovação em todos os testes do NIST. Portanto, se for utilizada uma taxa maior que a taxa máxima ocorre uma degradação nas proprieadades estatísticas das sequências binárias e em consequência a reprovação no NIST.

Calculando a média para os PRNG baseados na $q$ deformação e no mapa de Arnold a partir das proporções indicadas na Tabela I, obtemos 0,9834 e 0,9836, respectivamente, indicando que os dois PRNG possuem uma média de aprovação equivalente. Desta forma, ambos os PRNG conseguem gerar sequências binárias com boas propriedades estatísticas. Entretanto, o PRNG proposto neste trabalho possui taxa superior, que é taxa máxima permitida $r=1$, resultando em uma maior capacidade de geração de bits para conjuntos discretos com cardinalidade equivalente, em comparação com o PRNG proposto em [10].

Uma outra comparação pode ser feita com um PRNG baseado em mapas caóticos reais. Em [15] é proposto um PRNG baseado no mapa logístico sobre os números reais. No referido trabalho, é empregado o mapa logístico com parâmetro de controle variável com o objetivo de melhorar a estatística das sequências binárias obtidas. Cada amostra caótica gerada pela iteração do mapa logístico é representada com 32 bits e desta representação binária extrai-se o bit menos significativo, e taxa resultante é $r=1 / 32$. Para avaliar a qualidade das sequências binárias obtidas em [15], também é utlizada a suíte NIST e as sequências passam em todos os testes com uma média de aprovação de 0,989 . Esta média é um pouco superior à taxa obtida pelo PRNG proposto neste trabalho, entretanto o PRNG proposto possui uma maior capacidade de geração de bits por amostra devido à sua taxa superior.

\section{CONClusões}

Neste trabalho definimos e analisamos algumas propriedades algébricas de $q$-análogos em corpos finitos $\mathbb{Z}_{p}$. Estabelecemos as condições necessárias para as quais a $q$-deformação é uma permutação dos elementos de $\mathbb{Z}_{p}$. Apresentamos uma possível aplicação dos $q$-análogos como um PRNG e simulações numéricas utilizando a suíte estatística NIST evidenciam que o PRNG proposto possui boas propriedades estatísticas. Além disso, o PRNG proposto possui taxa máxima de geração de bits, enquanto na literatura, em geral, são consideradas taxas baixas para a obtenção de boas propriedades estatísticas.

Um prosseguimento natural deste trabalho, atualmente em andamento pelos autores, é a definição de $q$-análogos em corpos de extensão $\operatorname{GF}\left(p^{k}\right)$, bem como a busca por novas aplicações de $q$-análogos em corpos finitos. Uma possibilidade de aplicação para os $q$-análogos é utilizar a dinâmica iterativa gerada pela aplicação sucessiva da $q$-deformação em processos de cifragem, em que os parâmetros $p$ e $q$ podem ser considerados chaves secretas. Para valores elevados de $p$ tornase inviável buscar todos os valores de $q$ que são geradores de $G$ e em seguida gerar todas as permutações de $\mathbb{Z}_{p}$ para todos os valores possíveis de $q$ para realizar uma busca por força bruta. No caso do cálculo da transformação inversa, o problema recai em um problema de logaritmo discreto, que em geral é um problema computacionalmente inviável.

Ainda em consequência dos resultados apresentados neste trabalho, um outro prosseguimento, também em andamento pelos autores, é investigar o efeito da $q$-deformação em sistemas definidos em estruturas discretas, como por exemplo mapas caóticos discretos. Alguns trabalhos recentes analisam o efeito da $q$-deformação na dinâmica de mapas caóticos reais conhecidos, como o mapa logístico e o mapa de Hénon. Entretanto, no caso de mapas caóticos discretos, ainda não existem análises do efeito da $q$-deformação na dinâmica destes mapas e nas suas propriedades estatísticas.

\section{REFERÊNCIAS}

[1] P. C. Victor Kac, Quantum calculus, 1st ed., ser. Universitext. SpringerVerlag, New York, 2002.

[2] R. R. George E. Andrews, Richard Askey, Special functions, ser. Encyclopedia of mathematics and its applications 71. Cambridge University Press, 1999

[3] S. Morier-Genoud and V. Ovsienko, "On q-deformed real numbers," Experimental Mathematics, p. 1-9, Oct. 2019.

[4] - "q-deformed rationals and q-continued fractions," Forum of Mathematics, Sigma, vol. 8, March 2020.

[5] V. Patidar, G. Purohit, and K. K. Sud, "Dynamical behavior of qdeformed Henon map," International Journal of Bifurcation and Chaos, vol. 21, no. 05, pp. 1349-1356, July 2011.

[6] S. Behnia, M. Yahyavi, and R. Habibpourbisafar, "Watermarking based on discrete wavelet transform and q-deformed chaotic map," Chaos, Solitons \& Fractals, vol. 104, pp. 6-17, Nov. 2017.

[7] J. Cánovas and M. Muñoz-Guillermo, "On the dynamics of the qdeformed logistic map,” Physics Letters A, vol. 383, no. 15, pp. 17421754, may 2019.

[8] G.-C. Wu, M. Niyazi Çankaya, and S. Banerjee, "Fractional q-deformed chaotic maps: A weight function approach," Chaos: An Interdisciplinary Journal of Nonlinear Science, vol. 30, no. 12, p. 121106, Dec. 2020. 
[9] L. Kocarev, J. Szczepanski, J. M. Amigo, and I. Tomovski, "Discrete chaos-I: Theory," IEEE Trans. Circuits Syst. I: Reg. Papers, vol. 53, no. 6, pp. 1300-1309, June 2006.

[10] C. E. C. Souza, D. P. B. Chaves, and C. Pimentel, "One-dimensional pseudo-chaotic sequences based on the discrete Arnold's cat map over Z3m," IEEE Transactions on Circuits and Systems II: Express Briefs, vol. 68, no. 1, pp. 491-495, Jan. 2021.

[11] B. Chirikov and F. Vivaldi, "An algorithmic view of pseudochaos," Physica D: Nonlinear Phenomena, vol. 129, no. 3, pp. 223 - 235, May 1999.

[12] L. E. B. III et al., SP 800-22 Rev. 1a. A Statistical Test Suite for Random and Pseudorandom Number Generators for Cryptographic Applications. Gaithersburg, MD, United States: Nat. Inst. Std. \& Technol., 2010.

[13] R. J. McEliece, Finite fields for computer scientists and engineers, 1st ed., ser. The Kluwer international series in engineering and computer science Information theory SECS23. Kluwer Academic Publishers, 1987.

[14] G. H. Hardy, E. M. Wright, D. R. Heath-Brown, and J. H. Silverman, An Introduction to the Theory of Numbers, 6th ed. Oxford University Press, 2008

[15] M. Garcia-Bosque, A. Pérez-Resa, C. Sánchez-Azqueta, C. Aldea, and S. Celma, "Chaos-based bitwise dynamical pseudorandom number generator on FPGA," IEEE Transactions on Instrumentation and Measurement, vol. 68, no. 1, pp. 291-293, Jan. 2019.

[16] R. Lan, J. He, S. Wang, Y. Liu, and X. Luo, "A parameter-selectionbased chaotic system," IEEE Transactions on Circuits and Systems II: Express Briefs, vol. 66, no. 3, pp. 492-496, March 2019. 